\title{
Nootropic Effect of Fenugreek Seed Extract against Scopolamine Induced Cognitive
}

\section{Decline in Experimental Mice}

4 Shalam Mohamed Hussain ${ }^{1 *}$, Nayef Almutairi², Fahad Alrakaf ${ }^{2}$, Mohammed Aljameli ${ }^{2}$,

5 Muhammad Alshammari ${ }^{3}$ and Sulaiman Alnasser ${ }^{4}$

61 Former faculty and independent researcher, College of Pharmacy, Qassim University, Saudi Arabia

2 Graduate students, Unaizah College of Pharmacy, Qassim University, Saudi Arabia

${ }^{3}$ Department of Pharmacy Practice, Unaizah College of Pharmacy, Qassim University, Saudi Arabia

4 Department of Pharmacology, Unaizah College of Pharmacy, Qassim University, Saudi Arabia

*Correspondence: shalam26@yahoo.co.in

Abstract: Background: Alzheimer's disease affecting about 24 million people world-wide. The socio-economic burden on world-economies costing more than 172 billion US \$ annually for the US alone. Objectives: To prepare aqueous extract of $\mathrm{T}$. foenum graecum seeds (FSE) to explore the possible treatment for cognitive deficit in experimental animals. Materials and methods: FSE was subjected to preliminary phytochemical evaluation and antioxidant effect using free radical scavenging method (DPPH). All the animal behavior was video recorded with no human intervention during observation and animal groupings were blinded to avoid investigator bias. Different doses of FSE (5\%, 10\% and 20\%), control, standard (Piracetam, $200 \mathrm{mg} / \mathrm{kg}$, IP.) were given for male albino mice a period of 15 days followed by cognitive assessment in elevated plus maze and novel objection recognition tests. Ttransfer latencies and time exploring novel and familiar objects were recorded in respective tests. Retention of this learned-task was examined again $24 \mathrm{~h}$ later and inflexion ratio (IR) and discriminative index (DI) were calculated respectively. Next in the second set of experiment same groups and treatments were continued but scopolamine was administered to all the groups except normal control one hour after the last dose and examined similarly. Results: FSE showed potential antioxidant effect and a dose dependent increase in transfer latency and improved DI indicating a nootropic effect. FSE at 20\% showed significant reversal of scopolamine induced dementia in the second set of experiment. Conclusion: FSE improved memory as well as reversed the chemically induced memory deficits in experimental mice. 


\section{Introduction}

Alzheimer's disease (AD) is a neurodegenerative disorder that it is the most common cause of dementia in the old age who are slowly deprived in memory and the ability to carry out the simple tasks. People with AD tend to lose their cognitive skills, including behavioral disabilities and loss of functional autonomy. Both genetic and environmental factors are known to be an AD risk factor. Free radicals, elevated oxidative stress and mitochondrial dysfunction, eventually triggering neuronal / synaptic and neurodegenerative dysfunction ${ }^{1}$. According to the National Institute of Health, some 18 million people worldwide have been affected and are estimated to rise by 33 to around 65.7 million by 2030 and 115.4 million by 34 by $2050^{2}$. The Alzheimer's Disease Association estimated that Alzheimer's disease accounts for 50 to 80 per cent of cases of dementia worldwide, with the largest identified risk factor rising the age of 65 and older and the prevalence rate of Alzheimer's disease was not documented yet ${ }^{1}{ }^{3}$. Several drugs, such as rivastigmine and donepezil, are used to treat this condition as inhibitors of Acetyl-cholinesterase (AChE) licensed by a variety of global food and drug companies. ${ }^{4}$ Despite the use of these inhibitors to control the role of AChE, there is a growing need to seek new medications. Therefore, several studies for this reason were aimed at new natural compounds with potential antioxidant properties and with very low side effects have been reported. As a consequence, the use of imitative herbal medicines for $\mathrm{AD}$ treatment is on the increase. As is already known, acetylcholine is the key neurotransmitter that plays a vital role in AD. For this reason, several trials have been performed to use AChE suppressors. 5, 6,7

Fenugreek is one of the most important plants with antioxidant properties. Its seeds and leaves are used for food and also in traditional medicines. Some studies stated that trigonelline, a compound isolated from fenugreek, exhibited nerve regeneration and enhanced memory activity in AD-induced mice. Its seeds and leaves are used in food and herbal medicine as well. Fenugreek seeds have been found to have a variety of compounds, such as steroidal. The seeds of which are rich in choline, alkaloid, flavonoid, polyphenol antioxidants and other hydroxy-aromatic components which help it in exhibiting anti-oxidant, anti-inflammatory and neuro-protective properties. Therefore, the purported efficacy of this herb in in enhancing cognition was explored in the current study. ${ }^{8-12}$ (Figure 1)

\section{Materials and Methods}

\subsection{Drugs and chemicals}

Piracetam (Nootropil injection, a commercial product), standard nooropic agent. Commercially available scopolamine hydrobromide was purchased froma local pharmacy. Acetylthiocholine iodide, 5, 5'-dithiobis-2-nitrobenzoic acid, DPPH (2, 2, diphenyl-1-picryl hydrazil radical) was procured from Fluka Chemie (Buchs, Switzerland). All the other chemical agents used were of analytical grade available in our chemistry lab.

\subsection{Animals}

Male Swiss albino mice were procured from the commercial supplier and breeder in Riyadh, Saudi Arabia. Animal studies were performed after obtaining necessary permission from Institutional Animal Ethics Committee (IAEC UCP/18-19/01). After procuring the mice, they were acclimatized for 7 days and housed in groups of six under standard laboratory condition with relative humidity of $45-55 \%$ and light/ dark cycle of 12 hours. They were fed with synthetic standard pellet diet available locally and were supplied water ad libitum. Male mice weighing between 25-35 gm were used in this study and were fasted for $3 \mathrm{hrs}$ prior to any administration of vehicle/standard/extract. All the experimental procedures were carried out as per the protocol in a dimly lit room during the light period (8:00 to 16:00 hour). 


\subsubsection{Preparation of fenugreek seed extract}

The fresh Fenugreek seeds were purchased locally. The seeds were washed with tap water and dried in the shade at room temperature for 2 days. Then the dried seeds were kept for germination for 1 day in a covered muslin cloth. The germinated seeds were dried and powdered and sieved. Different concentrations $(5 \%, 10 \%$ and $20 \% \mathrm{w} / \mathrm{v})$ were prepared in water and stirred for two hours in magnetic stirrer and then centrifuged for 1 hour at 5000rpm. Supernatant was collected for administration.

\subsubsection{Determination of antioxidant activity of FSE}

Method followed as per the available references and consists of taking $3 \mathrm{ml}$ of $0.05 \mathrm{mM}$ DPPH in methanol with $30 \mu \mathrm{L}$ of the different concentrations of the extract in phosphate buffer (pH 7.4), mixed well and kept in dark for $20 \mathrm{~min}$ followed by reading absorbance. Blank reading was taken for DPPH alone without any extract. IC50 values for the different extracts were calculated and percentage inhibition was calculated as,

$\%$ inhibition $=[(\mathrm{Ab}-\mathrm{As}) / \mathrm{Ab}]{ }^{*} \mathbf{1 0 0}$, where $\mathrm{Ab}$ is control absorbance, As- sample absorbance. Vitamin $C 1 \mathrm{mM}$ and $1 \mathrm{mM}$ vitamin $\mathrm{E}$ were used as positive control ${ }^{13,14}$.

\subsubsection{Acute toxicity test}

Acute toxicity test of fenugreek seed extract was carried as per the method described in OECD Test Guidelines 425 (Up and Down Procedure) ${ }^{15}$ wherein a single albino mouse was given $2000 \mathrm{mg} / \mathrm{kg}$ p.o. as single dose and observed for first $30 \mathrm{~min}$, then for $4 \mathrm{~h}$. After survival of treated mouse, 4 additional mice were administered with the same dose under same conditions. Observed for 2 days for any signs of toxicity or death.

\subsubsection{Assessment of cognitive performance}

For all experimental procedures, all groups of treatments were blinded to the investigators to avoid any bias. The apparatus used for testing were cleaned with $5 \%$ alcohol before using each mouse to remove any animal cues. All the experiments were conducted in dim light and were video recorded for offline analysis

\subsubsection{Animal groupings}

Mice were divided into following groups each containing six, group I: Control (Distilled water $10 \mathrm{ml} / \mathrm{kg}$, p.o.), group II: Standard (Piracetam, $200 \mathrm{mg} / \mathrm{kg}$, IP.), group III: Low dose of FSE (5\%), po Group IV: Medium dose of FSE (10\%, po), Group V: High dose of FSE $(20 \%$, p.o). They were fasted for $3 \mathrm{~h}$ prior to the administration but water was supplied ad libitum. All the groups of mice were administered respective treatment as shown in the protocol (Figure 2).

\subsubsection{Elevated plus-maze}

The Elevated Plus maze (EPM) used was fabricated locally with wood and dimensions meeting the published literature. It had two open arms and two closed arms, crisscrossing each other forming a plus. The closed arms and open arms were $25 \mathrm{~cm} \times 10 \mathrm{~cm} \times 20 \mathrm{~cm}$ and $25 \mathrm{~cm} \times 10 \mathrm{~cm}$ respectively with a central platform of $10 \times 10 \mathrm{~cm}$ area. The entire maze was elevated to a height of $90 \mathrm{~cm}$ with a wooden column. All procedures were conducted in a dimly lit dark room $^{16-18}$. All the parameters were recorded using a web cam fixed above EPM to the roof and connected to a computer for recording and offline analysis. On day 15, $1 \mathrm{~h}$ after the dose, each mouse was placed at the end of an open arm, facing away from the central platform. Transfer latency (TL) i.e. the time taken by mouse with all its four legs to move into one of the enclosed 
120

121

122

123

124

125

126

127

128

129

130

131

132

133

134

135

136

137

138

139

140

141

142

143

144

145

146

147

148

149

150

151

152

153

154

arms was recorded as the initial transfer latency (L1) on the first day. If the animal does not enter into one of the enclosed arms within $90 \mathrm{~s}$, it was gently pushed into one of the two enclosed arms and the TL was assigned as $90 \mathrm{~s}$. The mouse was allowed to explore the maze for next $10 \mathrm{~s}$ and then returned to its home cage. Retention of this learned-task was examined again $24 \mathrm{~h}$ later (L2). The whole apparatus was thoroughly cleaned with $5 \%$ alcohol before placing each animal in the maze to avoid animal cues. The inflexion ratio (IR) was calculated by the following formula, $(\mathrm{IR})=(L 2-L 1) / L 1$, Where $L 1$ is the initial TL (s) on 1st day and L2 is the TL (s) on the 2nd day.

\subsubsection{Novel object recognition task ${ }^{19-21}$}

The apparatus was made up of wood of a rectangular box measuring $50 \mathrm{~cm} \times 50 \mathrm{~cm} \times 50 \mathrm{~cm}$. It was placed in dimly lit dark room. All the parameters were recorded using a video camera. Mice were divided into following groups each containing six. They were fasted for 3 hrs prior to the administration but water was supplied ad libitum. On day $17,1 \mathrm{~h}$ after the dose, each mouse was tested in a $30 \mathrm{~cm} \times 30 \mathrm{~cm}$ rectangular box. The test consists of 3 phases, 1 . habituation session 2 . training session 3. test session. All animals were given one habituation session in which they were allowed to explore the apparatus (without objects) for $10 \mathrm{~min}$. For the training session, each mouse was placed into the box with two identical objects ( 1 and 2 ) and allowed to explore for 5 min (training). The time spent by the animal exploring each object and also the time spent by the animal exploring both objects and the box were measured. $24 \mathrm{~h}$ after the training, one of the objects was replaced with a novel object (object number 3 , novel) and the other object is same as used for training (1, familiar object). Each mouse was individually tested and video graphed for 5 minutes. Time spent by mouse exploring objects determined.

2.3.4.4 Scopolamine induced amnesia in mice using above tests

In the second set of experiment same groups and treatment period were maintained but scopolamine ( $1 \mathrm{mg} / \mathrm{kg}$, IP) was administered to all the groups one hour after last dose on day 19 in the respective tests (EPM and NORT) and then examined to record as above. Retention of this learned-task was examined again $24 \mathrm{~h}$ later and parameters were calculated as per the procedure in above respective methods.

\subsection{Statistical Analysis}

All the results were expressed as mean \pm standard error. The data were analyzed using ANOVA followed by tukey's multiple comparison post hoc test. $p<0.05$ were considered as significant. The statistical analysis was done using the SPSS software package for Windows, version 20, Chicago, USA.

\section{Results}

\subsection{Antioxidant activity of FSE}

The decrease in DPPH absorption in the presence of varying concentrations of extract was monitored and it was noticed that the extract showed a dose dependent decrease in the absorbance of DPPH radical. IC50 value for the extract was found to be $9.93 \mu \mathrm{g} / \mathrm{ml}$. These results indicated an antioxidant potential of seed. (Table 1)

\subsection{Effect of FSE on transfer latency (TL) in elevated plus maze}

Effect of FSE on TL in mice were recorded with elevated plus maze apparatus where piracetam $200 \mathrm{mg} / \mathrm{kg}$ and FSE with three different dose levels (w/v) i.e. 5\%,10\% and 20\%, treated groups have shown a decrease in 
transfer latencies leading to corresponding increase in inflexion ratios as compared to normal control. But statistically significant effect $(P<0.05)$ was observed with high dose $20 \%$ of FSE and piracetam $(P<0.05)$. (Figure 3)

\subsection{Effect of FSE on transfer latency in scopolamine induced amnesic mice in EPM}

The effect of the vehicle, scopolamine $(1 \mathrm{mg} / \mathrm{kg})$, FSE $(5 \%, 10 \%$ and $20 \%)$ and piracetam (200 $\mathrm{mg} / \mathrm{kg})$ are shown in Figure 4. The scopolamine alone treated group showed a significant $(P<0.01)$ increase in TL values on the acquisition as well as on the retention days (decrease in inflexion ratio) as compared to vehicle control mice, indicating an impairment in learning and memory. Whereas in the acquisition as well as retention trial FSE demonstrated dose dependent decrease in the TL (increase in inflexion ratio) when compared to the scopolamine alone treated group $(P<0.01)$. Piracetam $(200 \mathrm{mg} / \mathrm{kg}$ IP.) exhibited marked decrease $(P<0.01)$ in TL in comparison with the scopolamine. However, FSE at the dose levels $20 \%$ and $10 \%$ showed a comparable decrease in the TL $(P<0.05)$.

\subsection{Effect of FSE for object exploration in mice using novel object recognition test}

Effect of FSE on inflexion ratios in mice were recorded with elevated plus maze apparatus. Piracetam 200 $\mathrm{mg} / \mathrm{kg}$ and FSE with three different dose levels i.e. 100, 200 and $400 \mathrm{mg} / \mathrm{kg}$, treated groups have shown decrease in transfer latencies leading to increase in inflexion ratios when compared to control. But statistically significant effect $(P<0.05)$ was observed with high doses i.e. 10 and $20 \%$ of FSE groups only indicating a dose dependent nootropic like effect. Piracetam also has increased the inflexion ratio very significantly $(P<$ 0.01). (Figure 5)

\subsection{Effect of FSE on time spent exploring in scopolamine induced dementia in mice using NORT}

The effect of the vehicle, scopolamine (1 $\mathrm{mg} / \mathrm{kg}, \mathrm{po})$, FSE (5\%,10\% and 20\%) and piracetam (200 $\mathrm{mg} / \mathrm{kg})$ were evaluated at the end of treatment period. The scopolamine $(1 \mathrm{mg} / \mathrm{kg})$ control group showed a significant $(P<0.01)$ increase in exploration time for novel object on the acquisition as well as on the retention days (decrease in discrimination index) as compared to vehicle control mice, indicating an impairment in learning and memory. In the acquisition as well as retention trial, FSE demonstrated dose dependent decrease in the exploration time (increase in DI) as compared to the scopolamine control group. Piracetam (200 mg/kg IP.) exhibited marked decrease $(P<0.01)$ in exploration time in comparison with the scopolamine control group. (Figure 6)

\section{Discussion}

Alzheimer's disease is a neurogenerative condition associated with a decrease in cognitive ability. ${ }^{4}$ Given the seriousness and high prevalence of this disease, the allopathic medical system has failed to provide a suitable cure. ${ }^{22}$ The present study therefore concentrated on investigating the memory enhancing function of the FSE in a chemical-induced amnesia models. In this study the exteroceptive model was used for evaluating the nootropic activity (memory enhancing) of FSE on learning and memory processes, which was indicated by decreased transfer latency and increased inflexion ratio in EPM. The interoceptive models used were amnesia induced by scopolamine, which was indicated by prevention of fall in transfer latency and inflexion ratio in EPM $^{16-18}$. The present study suggests that FSE possesses memory enhancing activity in view of its decreased transfer latency and increased inflexion ratio in EPM. This suggests that the FSE has pronounced nootropic effect which was comparable to nootropil (standard) in the study. FSE also exhibited a facilitatory effect on the retention of memory in scopolamine induced amnesic mice.

Similarly, in another group of models used by NORT, scopolamine substantially increased the e xploration period suggesting that scopolamine induced cognitive impairment in this model. Pret reatment with different doses of FSE greatly increased the ability of the treated mice to identify 
novel artifacts. Administration of different doses of FSE led to enhancement in indices of memory in normal as well as scopolamine induced memory impaired mice in EPM as well as NORT tests in the present. It is well known that cholinergic neuronal systems play an important role in cognitive deficiencies associated with $A D$, aging and neurodegenerative diseases. 23,24 In our study, amnesia caused by scopolamine is evident from the results obtained and its reversal with the prior treatment of FSE indicating the activation of cholinergic system by FSE. Also, the ability of the FSE to scavenge the oxidative free radical and to prevent induced tissue damage by its potential antioxidant activity in the DPPH free radical scavenging assay contributes to its cognition enhancing effect. In addition, FSE has phenolic and flavonoid compounds which are proven antioxidants. There are also numerous studies on the antioxidant capacity of fenugreek seeds. ${ }^{25-27}$ These findings indicate that they have antioxidant ability to prevent chemically mediated memory deficits. As a consequence, it can be concluded from these findings that FSE may provide a potential advantage in the amelioration of Alzheimer's disease type memory loss due to its probable potential for activation of the cholinergic system and/or free radical scavenging capability that can provide neuroprotection in the prevention or management of this disease. The effects observed with FSE are in agreement with the previous published studies wherein fenugreek extract as well as its primary constituent, trigolline exhibited potential cognitive effect in various chemically induced cognitive deficit models such as. ${ }^{24,26-30}$

Although this research was not an exhaustive adventure to draw any conclusions, it is proof of our hypothesis. However, more studies are required to further investigate the potential effects of FSE on AChE in various parts of the brain, amyloid beta plaques, the role of other neurotransmitters such as glutamate, gamma aminobutyric acid (GABA) and catecholamines.

\section{Conclusions}

In this study, we concentrated on exploring FSE's ability to improve memory in laboratory mice as well as reversing chemically induced memory deficits in experimental mice. The results of the invitro studies have shown that FSE is an antioxidant and the results of the in vivo analysis have concluded that FSE has nootropic function in the absence of cognitive deficits and has also been effective in preventing chemically induced memory deficits in experimental mice. The mechanism by which FSE has shown these properties can be related to its antioxidant, neuroprotective properties, its choline content or activation of acetylcholine system in brain. In the light of above, it may be worthwhile to explore the potential of these seeds in the management of $\mathrm{AD}$ patients

Author Contributions:

"Conceptualization, SMH.; methodology, SMH.; software, MS and SN.; validation, NA, FA and MA.; formal analysis, SMH.; investigation, NA, FA and MA.; resources, MS and SN.; data curation, SMH.; writing-SMH.; writing-review and editing, SMH, MS and SN; supervision, SMH.; project administration, NA, FA and MA. All authors have read and agreed to the published version of the manuscript.

Funding: Please add: “This research received no external funding.

Acknowledgments:

Authords would like to thank administrative and technical support provide by thee college authorities

Conflicts of Interest: The authors declare no conflict of interest.

\section{References}

1. Alzheimer's Disease and Related Dementias | National Institute on Aging. https://www.nia.nih.gov/health/alzheimers. Accessed September 23, 2020. 
3. International D. World Alzheimer Report 2019: Attitudes to Dementia; World Alzheimer Report 2019: Attitudes to Dementia.

4. P.A. D, R.S. M, M. G, J.D. L, J. F. Alzheimer's disease clinical and research update for health care practitioners. J Aging Res. 2013;2013(Mci).

5. Kennedy D, Scholey A. The Psychopharmacology of European Herbs with Cognition-Enhancing Properties. Curr Pharm Des. 2006;12(35):4613-4623. doi:10.2174/138161206779010387

6. Kiefer D. An herbal mixture for enhanced memory. Integr Med Alert. 2019;22(3).

7. Fu LM, Li JT. A systematic review of single Chinese herbs for Alzheimer's disease treatment. Evidence-based Complement Altern Med. 2011;2011. doi:10.1093/ecam/nep136

8. Yadav UCS, Baquer NZ. Pharmacological effects of Trigonella foenum-graecum L. in health and disease. Pharm Biol. 2014;52(2):243-254. doi:10.3109/13880209.2013.826247

9. Zhou J, Chan L, Zhou S. Trigonelline: A Plant Alkaloid with Therapeutic Potential for Diabetes and Central Nervous System Disease. Vol 19.; 2012.

10. Al-Asadi JN. OPEN ACCESS Therapeutic Uses of Fenugreek (Trigonella Foenum-Graecum L.) OPEN ACCESS.

11. Zandi P, Basu SK, Khatibani LB, et al. Fenugreek (Trigonella foenum-graecum L.) seed: a review of physiological and biochemical properties and their genetic improvement. Acta Physiol Plant. 2015;37(1):1-14. doi:10.1007/s11738-014-1714-6

12. Panda S, Biswas S, Kar A. Trigonelline isolated from fenugreek seed protects against isoproterenol-induced myocardial injury through down-regulation of Hsp27 and $\alpha$ B-crystallin. Nutrition. 2013;29(11-12):1395-1403. doi:10.1016/j.nut.2013.05.006

13. Ho KY, Tsai CC, Chen CP, Huang JS, Lin CC. Screening of Brazilian plant extracts for antioxidant activity by the use of DPPH free radical method. Phyther Res. 2001;15(2):127-130. doi:10.1002/ptr.687

14. Sharma OP, Bhat TK. DPPH antioxidant assay revisited. Food Chem. 2009;113(4):1202-1205. doi:10.1016/j.foodchem.2008.08.008

15. OECD. OECD Guidelines for the Testing of Chemicals, Section 4, Test No. 425: Acute Oral Toxicity - Upand-Down Procedure. Guidel Test Chem. 2001;(December):26. doi:10.1787/9789264071049-en

16. Elevated Plus Maze for Mice I Protocol. https://www.jove.com/t/1088/elevated-plus-maze-for-mice. Accessed September 24, 2020.

17. Komada M, Takao K, Miyakawa T. Elevated plus maze for mice. J Vis Exp. 2008;(22):1088. doi:10.3791/1088

18. Dhingra D, Kumar V. Memory-enhancing activity of palmatine in mice using elevated plus maze and Morris water maze. Adv Pharmacol Sci. 2012;2012. doi:10.1155/2012/357368

19. Scopolamine-induced deficits in a two-trial object recogniti...: NeuroReport. https://journals.lww.com/neuroreport/Abstract/1997/03240/Scopolamine_induced_deficits_in_a_two_trial_obje ct.23.aspx. Accessed September 24, 2020.

20. Lueptow LM. Novel object recognition test for the investigation of learning and memory in mice. J Vis Exp. 2017;2017(126):e55718. doi:10.3791/55718 
21. Botton PH, Costa MS, Ardais AP, et al. Caffeine prevents disruption of memory consolidation in the inhibitory avoidance and novel object recognition tasks by scopolamine in adult mice. Behav Brain Res. 2010;214(2):254-259. doi:10.1016/j.bbr.2010.05.034

22. Schneider L, DeKosky S, Farlow M, Tariot P, Hoerr R, Kieser M. A Randomized, Double-Blind, PlaceboControlled Trial of Two Doses of Ginkgo Biloba Extract in Dementia of the Alzheimers Type. Curr Alzheimer Res. 2005;2(5):541-551. doi:10.2174/156720505774932287

23. Parsons CG, Danysz W, Dekundy A, Pulte I. Memantine and cholinesterase inhibitors: Complementary mechanisms in the treatment of Alzheimer's disease. Neurotox Res. 2013;24(3):358-369. doi:10.1007/s12640-0139398-z

24. Anjaneyulu K, Rai KS, Rajesh T, Nagamma T, Bhat KMR. Therapeutic efficacy of fenugreek extract or/and Choline with docosahexaenoic acid in attenuating learning and memory deficits in ovariectomized rats. J Krishna Inst Med Sci Univ. 2018;7(2):10-20.

25. Bafadam S, Beheshti F, Khodabakhshi T, et al. Trigonella foenum-graceum seed (Fenugreek) hydroalcoholic extract improved the oxidative stress status in a rat model of diabetes-induced memory impairment. Horm Mol Biol Clin Investig. 2019;39(2):1-13. doi:10.1515/hmbci-2018-0074

26. Moin M, Kassaee SM. Effect of Fenugreek on Memory and Learning and also Antioxidant Capacity in Hippocampus of Type II Diabetic Rats. J Ilam Univ Med Sci. 2017;25(4):13-23. doi:10.29252/sjimu.25.4.13

27. Khalili M, Alavi M, Esmaeil-Jamaat E, Baluchnejadmojarad T, Roghani M. Trigonelline mitigates lipopolysaccharide-induced learning and memory impairment in the rat due to its anti-oxidative and antiinflammatory effect. Int Immunopharmacol. 2018;61(September 2017):355-362. doi:10.1016/j.intimp.2018.06.019

28. Chowdhury AA, Gawali NB, Munshi R, Juvekar AR. Trigonelline insulates against oxidative stress, proinflammatory cytokines and restores BDNF levels in lipopolysaccharide induced cognitive impairment in adult mice. Metab Brain Dis. 2018;33(3):681-691. doi:10.1007/s11011-017-0147-5

29. Fahanik-Babaei J, Baluchnejadmojarad T, Nikbakht F, Roghani M. Trigonelline protects hippocampus against intracerebral A $\beta(1-40)$ as a model of Alzheimer's disease in the rat: insights into underlying mechanisms. Metab Brain Dis. 2019;34(1):191-201. doi:10.1007/s11011-018-0338-8

30. Prema A, Thenmozhi AJ, Manivasagam T, Essa MM, Akbar M, Akbar MD. Fenugreek seed powder nullified aluminium chloride induced memory loss, biochemical changes, A $\beta$ burden and apoptosis via regulating Akt/GSK3 $\beta$ signaling pathway. PLoS One. 2016;11(11):1-19. doi:10.1371/journal.pone.0165955 
320 1. Table

321 2. Table 1. Antioxidant activity of FSE using DPPH free radical scavenging activity

\begin{tabular}{|c|c|c|c|c|c|c|c|}
\hline 3. & S.No & 4. $\quad$ ( & $\begin{array}{l}\text { Concentration } \\
\text { of extract } \\
(\mu \mathrm{g} / \mathrm{ml} \quad \text { of } \\
\text { FSE) }\end{array}$ & 5. & $\begin{array}{l}\text { Inhibitory } \\
\text { activity (\%) }\end{array}$ & 6. & $\begin{array}{l}\text { IC50 } \\
\mu \mathrm{g} / \mathrm{ml}\end{array}$ \\
\hline 7. & 1 & 8. 1 & 10 & 9. & 50 & \multirow{10}{*}{10.} & \multirow{10}{*}{9.93} \\
\hline 11. & 2 & 12. & 20 & 13. & 83 & & \\
\hline 14. & & 15.3 & 30 & 16. & 85 & & \\
\hline 17. & 4 & 18. & 40 & 19. & 86 & & \\
\hline 20. & 5 & 21.5 & 50 & 22. & 86 & & \\
\hline 23. & 6 & 24. & 60 & 25. & 87 & & \\
\hline 26.7 & 7 & 27.7 & 70 & 28. & 89 & & \\
\hline 29. & 8 & 30.8 & 80 & 31. & 90 & & \\
\hline 32. & 9 & 33. & 90 & 34. & 90 & & \\
\hline 35. & 10 & 36.1 & 100 & 37. & 90 & & \\
\hline
\end{tabular}

38. 
$324 \quad 40 . \quad$ Figures

Figure 1 Image of leaves and seeds of Trigonella foenum grtecum and chemical structure of trigonelline

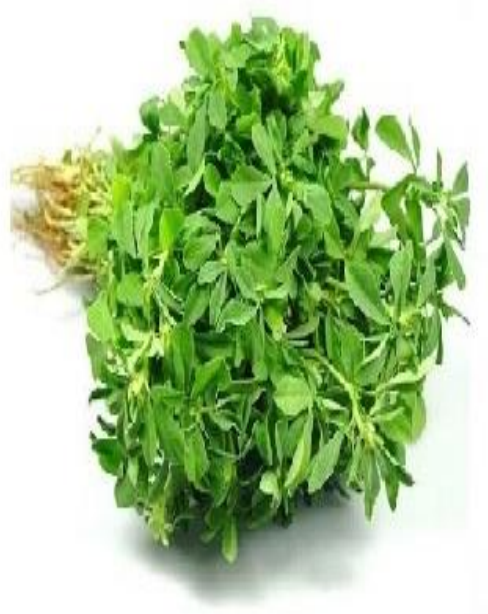

Leaves of Trigonella foenum graecum

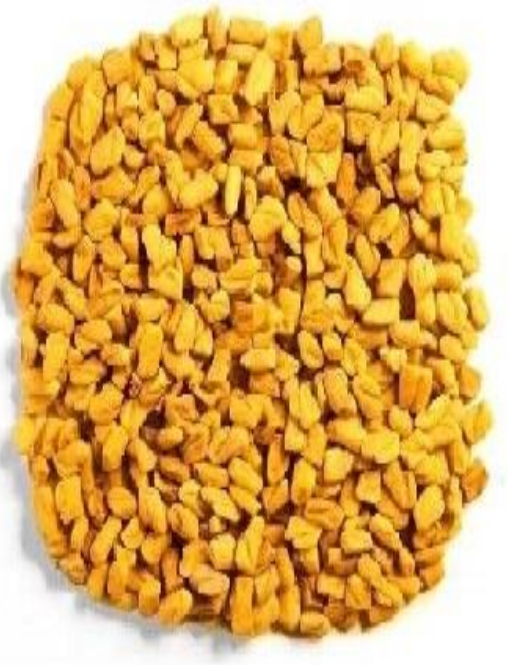

Seeds of Trigonella foen um graecum<smiles>C[n+]1cccc(C(=O)[O-])c1</smiles>

Chemical structure of trigonelline

$325 \quad 41$.

$326 \quad 42$. 
328

329

330

45.

33146.
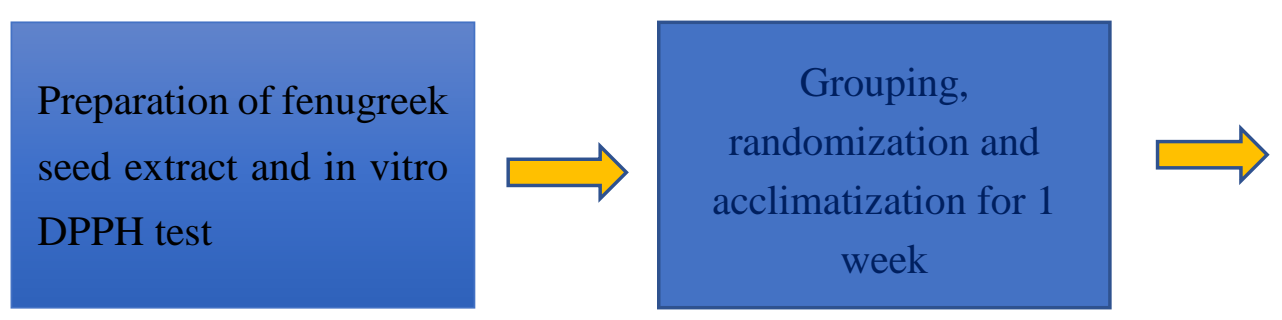

FSE $(5 \%, 10 \%$ and $20 \%$ in $10 \mathrm{ml} / \mathrm{kg}$, p.o.), Piracetam (200 $\mathrm{mg} / \mathrm{kg}$, p.o.) contd.

Day 0 - Day 15

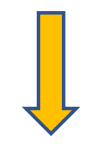

Scopolamine (1 $\mathrm{mg} / \mathrm{kg}$, IP) one hour after last dose. Repeated cognitive assessment in EPM and

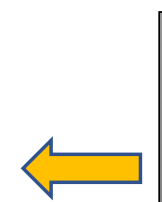

xinnm

Day 19-Day 20

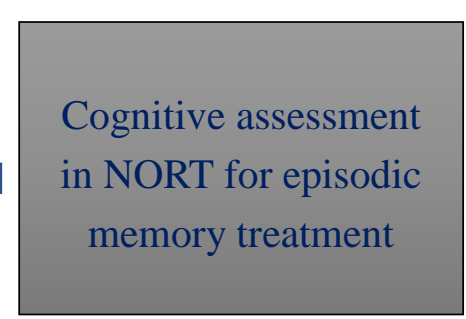

Day 17 - Day 18

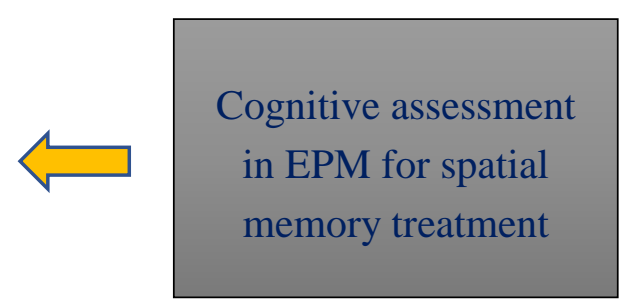

Day 15 - Day 16 
332 47. Fig 3: Effect of FSE on inflexion ratio in mice in elevated plus maze

33348.

$334 \quad 49$.

33550.

$336 \quad 51$.

$337 \quad 52$.

0.8

33853

0.7

33954.

54.

\begin{tabular}{ll}
0.6 \\
\hdashline
\end{tabular}

$340 \quad 55$.

范 0.4

34156.

34257

34358.

34459.

345

59.
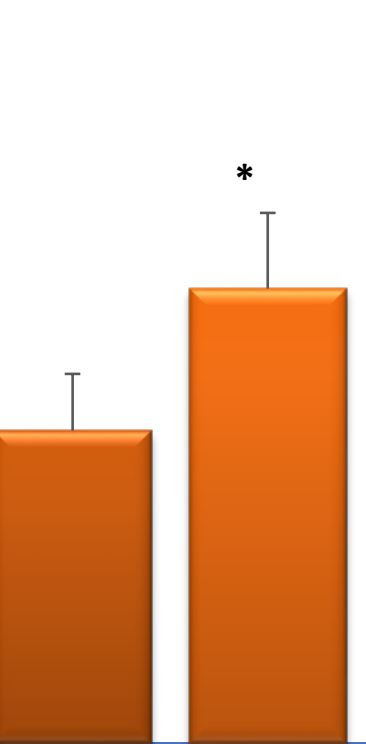

- Normal

control

口Piracetam

$\square \mathrm{FSE}(5 \%)$

$\square \mathrm{FSE}(10 \%)$

$\square \mathrm{FSE}(20 \%)$

346 
61. Figure 4: Effect of FSE on inflexion ratio in scopolamine induced amnesic mice in elevated plus maze

62.

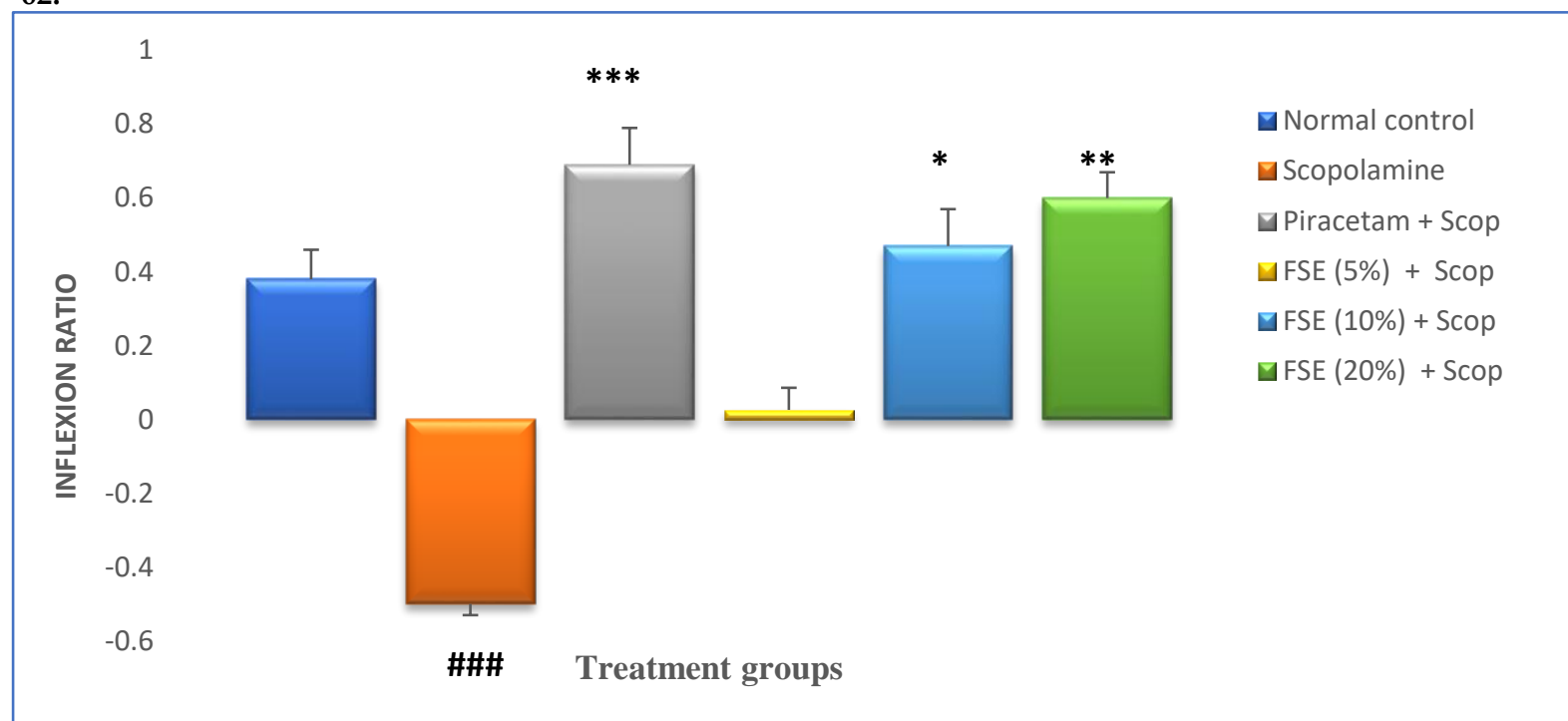

71. Statistical analysis by one-way ANOVA followed by Dunnett's't' test. Values are expressed as mean \pm S.E.M $(n=6) .{ }^{*} \mathrm{p}<0.05,{ }^{* *} \mathrm{p}<0.01$ compared with normal control group. \#\#p<0.01when compared with disease control (Scopolamine)

72.

73. Figure 5: Effect of FSE on discrimination index in mice in novel object recognition test
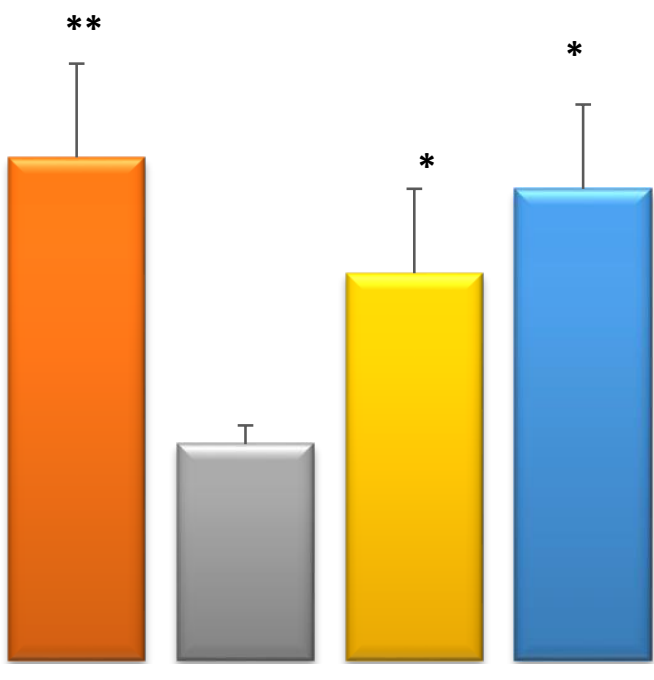

\Normal control

๑Piracetam

$\square$ FSE (5\%)

$\triangle \mathrm{FSE}(10 \%)$

口FSE (20\%)

\section{TREATMENT GROUPS}

84. Statistical analysis by one-way ANOVA followed by Dunnett's' test. Values are expressed as mean \pm S.E.M $(n=6) .{ }^{*} \mathrm{p}<0.05,{ }^{* *} \mathrm{p}<0.01$ compared with normal control group 
85. Figure 6: Effect of FSE on discrimination index in scopolamine treated mice in novel object recognition test

380

86.

\section{Discriminative Index}

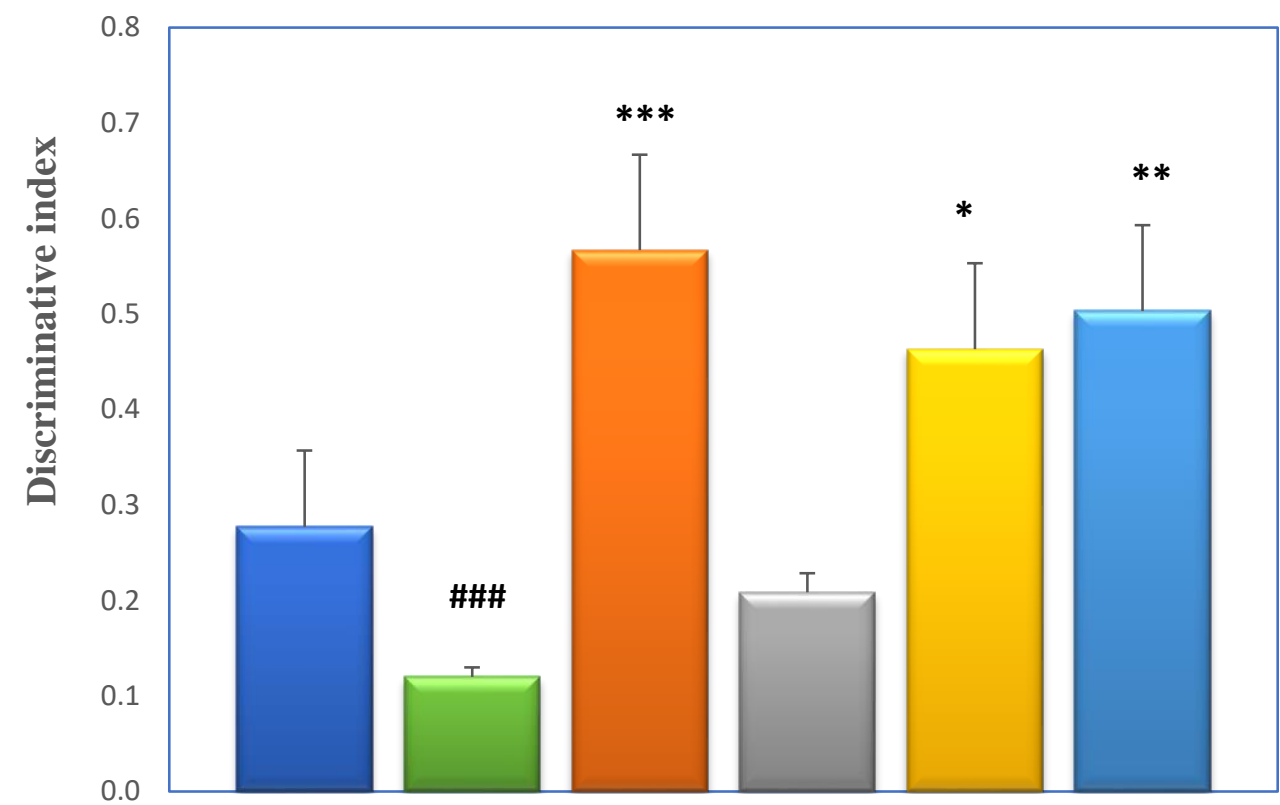

Q Normal control

$\square$ Scopolamine

Q Piracetam + Scop

$\square$ FSE (5\%) + Scop

$\triangle$ FSE $(10 \%)+$ Scop

$\square$ FSE $(20 \%)+$ Scop

\section{TREATMENT GROUPS}

89. Statistical analysis by one-way ANOVA followed by Dunnett's' test. Values are expressed as mean \pm S.E.M $(n=6) .{ }^{*} \mathrm{p}<0.05,{ }^{* *} \mathrm{p}<0.01$ compared with scopolamine control group. \#\#p<0.01when compared with normal control 